\title{
Differential effects of amount on temporal and probability discounting of gains and losses
}

\author{
SARA J. ESTLE, LEONARD GREEN, JOEL MYERSON, and DANIEL D. HOLT \\ Washington University, St. Louis, Missouri
}

\begin{abstract}
In four experiments, we compared the effects of delay, probability, and monetary amount on the subjective value of gains and losses. For delayed gains, smaller amounts were discounted more steeply than larger amounts, whereas the opposite pattern was observed with probabilistic gains. For both delayed and probabilistic losses, however, amount had much smaller and less reliable effects on discounting. Taken together, the pattern of differential magnitude effects leads to delayed gains' being discounted significantly more steeply than delayed losses, but only at smaller amounts, whereas probabilistic gains are discounted significantly more steeply than probabilistic losses, but only at larger amounts. Even though the same hyperbola-like function described both individual and group discounting of delayed and probabilistic gains and losses, the present findings suggest that different processes are involved in discounting positive and negative outcomes. Raw data may be downloaded from www.psychonomic.org/archive.
\end{abstract}

People (and nonhuman animals) generally prefer an immediate reward to a delayed reward of the same amount. Such preferences can be understood in terms of temporal discounting. That is, the relatively greater subjective value of the immediate reward may be assumed to reflect the fact that the value of the delayed reward is discounted. Similarly, a certain reward generally is preferred to an uncertain or probabilistic reward of the same amount, and such preferences may be understood in terms of probability discounting (for a review, see Green \& Myerson, 2004).

Temporal and probability discounting are involved in many instances of daily decision making (e.g., a choice between spending now or investing for the future). Of course, daily decision making involves not only positive outcomes, such as rewards, but also negative outcomes. When shopping, for example, people are often confronted with a choice between paying for an item immediately or making a delayed payment that would include interest charges in addition to the purchase price. If delayed losses (e.g., deferred payments) are discounted in a fashion similar to that for delayed gains, people might prefer larger delayed losses over smaller immediate ones. The extensive credit card debt in the U.S., which corresponds to approximately $\$ 6,500$ per household, ${ }^{1}$ appears to exemplify this tendency toward discounting delayed losses. Despite its obvious importance, the discounting of losses has received much less experimental attention than has the discounting of gains.

A preliminary report of this study was presented at the May 2003 meeting of the Society for Quantitative Analyses of Behavior in San Francisco. Support for this research was provided by Grant MH55308 from the National Institute of Mental Health. We thank Joe Donlin for his help in running the first two experiments. S.J.E. and D.D.H. are now at the University of Wisconsin, Eau Claire. Correspondence concerning this article should be addressed to L. Green, Department of Psychology, Campus Box 1125, Washington University, St. Louis, MO 63130 (e-mail: lgreen@wustl.edu).
Various equations (i.e., discounting functions) have been proposed to describe the decrease in subjective value of a delayed or probabilistic reward with increases in the delay until, or the odds against, receiving the reward (see, e.g., Kirby, 1997; Loewenstein \& Prelec, 1992; Myerson \& Green, 1995; Raineri \& Rachlin, 1993). Determining the form of the discounting function is important because different equations instantiate different assumptions regarding some fundamental aspects of the choice process.

For example, an exponential temporal discounting function would be consistent with standard microeconomic theory and the assumption of a purely rational decision maker (for a historical review, see Loewenstein, 1992). Further, exponential discounting assumes that there is an opportunity cost in waiting for delayed rewards, because waiting means forgoing what could be earned by investing the immediate reward, and/or because waiting for a delayed reward involves risks such that with each additional unit of waiting time there is a constant probability that something will happen to prevent the reward's receipt (Green \& Myerson, 1996).

There is, however, a growing consensus that, compared with an exponential function, a hyperbola-like function provides a much better description of the relation between subjective value and the delay until receiving a reward (e.g., Ainslie, 1992; Green, Fry, \& Myerson, 1994; Kirby, 1997; Laibson, 1997; Mazur, 1987; Myerson \& Green, 1995; Rachlin, Raineri, \& Cross, 1991; for reviews of the economic and psychological literatures on temporal discounting, see Frederick, Loewenstein, \& O'Donoghue, 2002; Green \& Myerson, 2004). For delayed rewards, the discounting function is given by

$$
V=A /(1+b X)^{s},
$$

where $V$ represents the subjective value of a delayed reward, $A$ represents its amount, $b$ is a parameter governing 
the rate of discounting, $X$ is the delay, and $s$ is a parameter that represents the nonlinear scaling of amount and/or time (Stevens, 1957). When $s$ equals 1.0, the discounting function is a simple hyperbola. When $s$ is less than 1.0 , as is often the case, the discounting curve decreases less sharply at higher delay values than does a simple hyperbola with the same discounting rate parameter, $b$. For probabilistic rewards, a similar equation holds in which $X$ is the odds against receiving the reward (Green, Myerson, \& Ostaszewski, 1999; Rachlin et al., 1991), and the parameter $s$ may represent the nonlinear scaling of amount and/or the odds against an outcome.

In the case of temporal discounting, the hyperbola-like form of Equation 1 may be derived from the assumption that people make choices on the basis of comparing rates of reward, where both amount and time are scaled according to psychophysical power functions (Myerson \& Green, 1995). That is, the subjective value of a delayed reward is assumed to be proportional to the perceived rate of reward (i.e., the ratio of the psychophysically scaled amount of the reward to the psychophysically scaled duration of the delay until its receipt). According to the derivation of Equation 1 proposed by Myerson and Green, the parameter $s$ represents the ratio of the exponents for the power functions for delay and amount. Thus, when $s$ is less than 1.0 , as is often observed, it follows that the exponent for delay is less than the exponent for amount. Given that in both cases the psychophysical power functions are negatively accelerated (as indicated in the case of monetary rewards by the principle of diminishing marginal returns), the implication is that scaling of amount is closer to linearity than is the scaling of time.

In the case of probability discounting, Equation 1 may be derived by assuming that the odds against receiving a reward also are scaled according to a power function (Green \& Myerson, 2004). Because the exponent for empirical probability discounting functions typically is less than the exponent for empirical temporal discounting functions (see, e.g., Green et al., 1999; Myerson, Green, Hanson, Holt, \& Estle, 2003), the implication is that the scaling of odds is even more negatively accelerated than the scaling of time, although this assumption has not been directly tested.

Alternatively, Prelec and Loewenstein (1991) have argued that the discounting function's hyperbola-like form is consistent with two hypothesized characteristics of comparison judgments, which they term increasing proportional sensitivity and decreasing absolute sensitivity. It should be noted that in their formulation of the probability discounting function, the $X$ variable represents the logarithm of 1 plus the odds against receiving the reward, rather than simply the odds against (Myerson et al., 2003).

Although many studies have examined the form of the temporal discounting function for rewards, only one experimental study has addressed the question of whether the same form of mathematical function describes the discounting of both delayed gains and losses (Murphy,
Vuchinich, \& Simpson, 2001). Murphy et al. replicated previous findings of a gain-loss asymmetry in which delayed gains are discounted more steeply than delayed losses (Benzion, Rapoport, \& Yagil, 1989; Thaler, 1981), and they reported that a simple hyperbola (i.e., Equation 1 with $s$ fixed at 1.0) described the temporal discounting of both gains and losses. However, recent studies of discounting involving delayed gains have demonstrated that, in many cases, $s$ is significantly less than 1.0 (e.g., Myerson \& Green, 1995; Myerson et al., 2003; Simpson \& Vuchinich, 2000), and the question arises as to whether this also is true for delayed losses. Moreover, despite a long tradition of research on decision making under risk and uncertainty (e.g., Kahneman \& Tversky, 1979; Starmer, 2000) that includes a number of studies examining the mathematical form of the function that describes the discounting of probabilistic gains (e.g., Gonzalez \& $\mathrm{Wu}, 1999)$, very few studies have examined the discounting function for probabilistic losses, and to the best of our knowledge, none has directly compared the probability discounting function for losses with that for gains.

Another important issue concerns the effect of amount on the rate at which delayed and probabilistic outcomes are discounted. There is now a general consensus that the discounting of gains is strongly affected by amount, and that amount of reward has opposite effects on temporal and probability discounting. Whereas smaller delayed rewards are discounted more steeply than larger delayed rewards (e.g., Chapman \& Elstein, 1995; Green, Myerson, \& McFadden, 1997; Kirby, 1997; Raineri \& Rachlin, 1993), smaller probabilistic rewards tend to be discounted less steeply than larger probabilistic rewards (Green et al., 1999; Myerson et al., 2003).

Although the effects of amount on the discounting of delayed and probabilistic gains are well established, the effects of amount on the discounting of delayed and probabilistic losses have received much less experimental attention. With respect to temporal discounting, there is some evidence of a magnitude effect with delayed losses, but it is unclear whether amount has a greater effect on the discounting of delayed gains than it does on the discounting of delayed losses. Baker, Johnson, and Bickel (2003) found a significant interaction between the amount and the sign of the outcome (i.e., gains vs. losses), whereas Chapman (1996) did not, and thus it remains an open question as to whether amount differentially affects the discounting of delayed gains and losses. With respect to probability discounting, as far as we know no study exists in which an interaction between the effects of the amount and sign of the outcomes on the degree of probability discounting has been sought.

Magnitude effects are of fundamental theoretical significance because they constitute an important anomaly with respect to standard microeconomic theory (Loewenstein \& Thaler, 1989). Moreover, the finding that amount has opposite effects on temporal and probability discounting of gains poses serious problems for theories that assume that the processes underlying decision making that 
involves delayed gains are the same as, or similar to, the processes underlying decision making that involves probabilistic gains (Green \& Myerson, 1996; Prelec \& Loewenstein 1991; Rachlin, Logue, Gibbon, \& Frankel, 1986; Rachlin, Siegel, \& Cross, 1994; Stevenson, 1986).

For example, in prospect theory (Kahneman \& Tversky, 1979) the discounting function (or, in economic terms, the utility function) for probabilistic outcomes is decomposed into a value function, which describes the relationship between the subjective value of a possible outcome and its amount, and a weighting function, which describes how the weight given to the subjective value of an outcome changes depending on its probability. Prelec and Loewenstein (1991) have extended this approach to delayed outcomes and localized the magnitude effect in the shape of the value function. If the magnitude effect is indeed localized in the value function, then the effect of amount on both delayed and probabilistic gains should be similar because the same value function applies in both cases (although, as already noted, amount of gain appears to affect temporal and probability discounting in opposite ways). Likewise, although the value function for losses differs from that for gains, the same value function should apply in decisions involving losses, regardless of whether they are delayed or probabilistic. Thus, the effects of amount on delayed and probabilistic losses also should be similar to each other. This latter prediction regarding the interaction of the effects of amount of loss and domain (temporal vs. probability discounting) has yet to be tested.

The overarching goal of the four experiments that make up the present study was to examine similarities and differences among the temporal and probability discounting of gains and losses, particularly with respect to the form(s) of the discounting function and the effects of amount on the gain-loss asymmetry in degree of discounting. For purposes of analytic clarity, all of the experiments were conducted within a discounting framework that incorporated parallel experimental procedures and the same statistical techniques. The discounting framework is well suited to reveal theoretically meaningful differences between temporal and probability discounting and between the discounting of gains and losses. In contrast, if different procedures and analytical techniques had been used to examine these phenomena, then any apparent differences in discounting could have been due to the procedural and analytic approaches rather than to true differences in the underlying processes (Green \& Myerson, 2004).

\section{EXPERIMENT 1}

In the first experiment, we addressed two basic questions regarding the discounting of delayed gains and losses. The first question was whether the mathematical function that best describes temporal discounting of gains (i.e., Equation 1) also describes temporal discounting of losses. Murphy et al. (2001) reported that a simple hyperbola (i.e., Equation 1 with $s=1.0$ ) described temporal discounting of both gains and losses. However, when Green and My- erson (2004) reanalyzed Murphy et al.'s data, they found that the proportion of variance explained by a hyperbolalike discounting function (i.e., Equation 1 with $s<1.0$ ) was significantly greater than that explained by the simple hyperbola, and that the values of the $s$ parameter were less than 0.3 for both gains and losses. The improvement in fit was most notable in the case of the discounting of delayed losses, for which the $R^{2}$ increased from .584 to .968 when the $s$ parameter was not constrained to equal 1.0. Green and Myerson reanalyzed only the group-level data from Murphy et al.'s experiment, however, and thus it is as yet unclear which form of discounting function best describes behavior at the individual level. It is well known that the form of the function fit to group averages can differ markedly from that which describes individual behavior (Estes, 1956; Sidman, 1952). Thus, a proper test of theoretical models of discounting must be based on individual data.

The second question addressed by this experiment was whether the magnitude effect observed in the temporal discounting of gains (i.e., greater discounting of smaller than of larger outcomes) also would be obtained with losses. To answer this question, we compared how steeply individuals discounted a smaller delayed amount (\$200) and a larger delayed amount $(\$ 40,000)$ when those amounts were gains and when they were losses.

\section{Method}

Participants. Twenty undergraduate students (7 males, 13 females; mean age $=19.5$ years) attending Washington University were recruited through the Department of Psychology's Human Subjects Pool and received course credit for their participation.

Procedure. The participants were tested individually in a small room containing a computer with a monitor and keyboard. The participants first were told that the purpose of the study was to examine their preferences for different hypothetical amounts of money, and then were administered the temporal discounting task. The task was divided into two phases, a gains phase and a losses phase, and the computer program randomly determined which of the two phases would be presented first. In addition, each phase was divided into two blocks, one for the smaller amount and one for the larger amount. The order in which the two amounts were studied was also randomly determined. The procedure for the temporal discounting task was similar to that used in previous studies of discounting (e.g., Du, Green, \& Myerson, 2002; Holt, Green, \& Myerson, 2003; Myerson et al., 2003).

The gains phase of the temporal discounting task began with the following instructions presented on the computer monitor.

In this part of the study, you will be asked to make several hypothetical decisions about different amounts of money that you might RECEIVE. We are interested in which amount you would choose to receive if you were to be offered these choices for real. Two amounts of money will appear on the screen. One amount can be received RIGHT NOW. The other amount can be received LATER, and the screen will show you how long you will have to wait until you receive this later amount. The RIGHT NOW amount of money will change after each of your decisions. The LATER amount of money will stay the same for a group of choices. Press the ' $Z$ ' key if you would prefer to RECEIVE the amount on the left of the screen. Press the ' $\mathrm{M}$ ' key if you would prefer to RECEIVE the amount on the right. THERE ARE NO CORRECT OR INCORRECT CHOICES. We are interested in which option you would really prefer. If you change your mind about a choice, you can return to the start of that group of choices by pressing the 'B' key.

These instructions were followed by six practice trials, after which any questions were answered and the experimental trials began. 
For each of the two delayed gain amounts ( $\$ 200$ and $\$ 40,000$ ), the participant made six choices at each of seven delays (1 month, 6 months, 1 year, 3 years, 5 years, 10 years, and 20 years) presented in increasing order. The first choice at each delay was between a delayed amount and an immediate amount equal to half of the delayed amount (e.g., \$200 in 1 month or $\$ 100$ now). For each of the subsequent choices at that delay, the amount of the immediate gain was adjusted on the basis of the participant's previous choice. If the participant chose the immediate gain, then its amount was decreased on the following trial; if the participant chose the larger, delayed gain, then the amount of the immediate gain on the next trial was increased.

The size of the adjustment to the immediate gain after the first choice was half of the smaller amount. For example, if the participant chose to receive $\$ 100$ now over $\$ 200$ in 1 month, then the alternatives on the next trial would be receiving $\$ 50$ now and $\$ 200$ in 1 month, whereas if the participant chose $\$ 200$ in 1 month on the first trial, then the alternatives on the next trial would be $\$ 150$ now and $\$ 200$ in 1 month. The size of the adjustment to the immediate gain decreased with each successive choice and was always equal to half of the previous adjustment, rounded to the nearest dollar. For example, if on the second trial the participant chose to receive $\$ 200$ in 1 month over \$50 now, then the alternatives on the third trial would be $\$ 75$ now and $\$ 200$ in 1 month. This iterative procedure converged upon an amount of an immediate gain that was close to the subjective value of the delayed gain.

If the participant chose the immediate gain on the last (sixth) trial, then the subjective value of the delayed gain was assumed to be slightly less than the immediate amount chosen, whereas if the participant chose the delayed gain on the last trial, the subjective value was assumed to be slightly more than the immediate amount that had been presented. More specifically, the subjective value was calculated as being halfway between the largest immediate amount that was preferred to the delayed amount and the smallest immediate amount that was preferred to the delayed amount. There were only two possible exceptions to this rule. If a participant always chose the delayed amount or always chose the immediate amount, then the subjective value was halfway between the last presented immediate amount and the maximum (i.e., the delayed amount; $\$ 200$ or $\$ 40,000$ ) or minimum (i.e., zero) amount, respectively.

The losses phase of the temporal discounting task began with instructions identical to those reported above except that the word RECEIVE was replaced with the word PAY. Again, the instructions were followed by six practice trials, and once the experimental trials began, the participants made a series of six choices at each of the same seven delays as in the gains phase. This was done for both the $\$ 200$ and $\$ 40,000$ amounts. The iterative procedure for the losses phase was similar to that for the gains phase, with the first adjustment equal to half of the immediate loss and each subsequent adjustment equal to half of the previous adjustment. The only difference was the direction of this adjustment.

For example, a participant's first choice in the smaller amount condition would be between paying $\$ 100$ now or paying $\$ 200$ in 1 month. If the participant chose to pay $\$ 100$ now, then the alternatives on the next trial would be paying $\$ 150$ now or $\$ 200$ in 1 month, whereas if the participant initially chose to pay $\$ 200$ in 1 month, then the alternatives on the next trial would be paying $\$ 50$ now or $\$ 200$ in 1 month. This iterative procedure converged upon an amount of an immediate loss that is close to the subjective value of the delayed loss. The subjective value of the delayed loss was calculated in a manner similar to that used to calculate the subjective value of the delayed gain.

\section{Results}

Figure 1 presents the group median subjective value plotted as a function of delay for both gains and losses. In
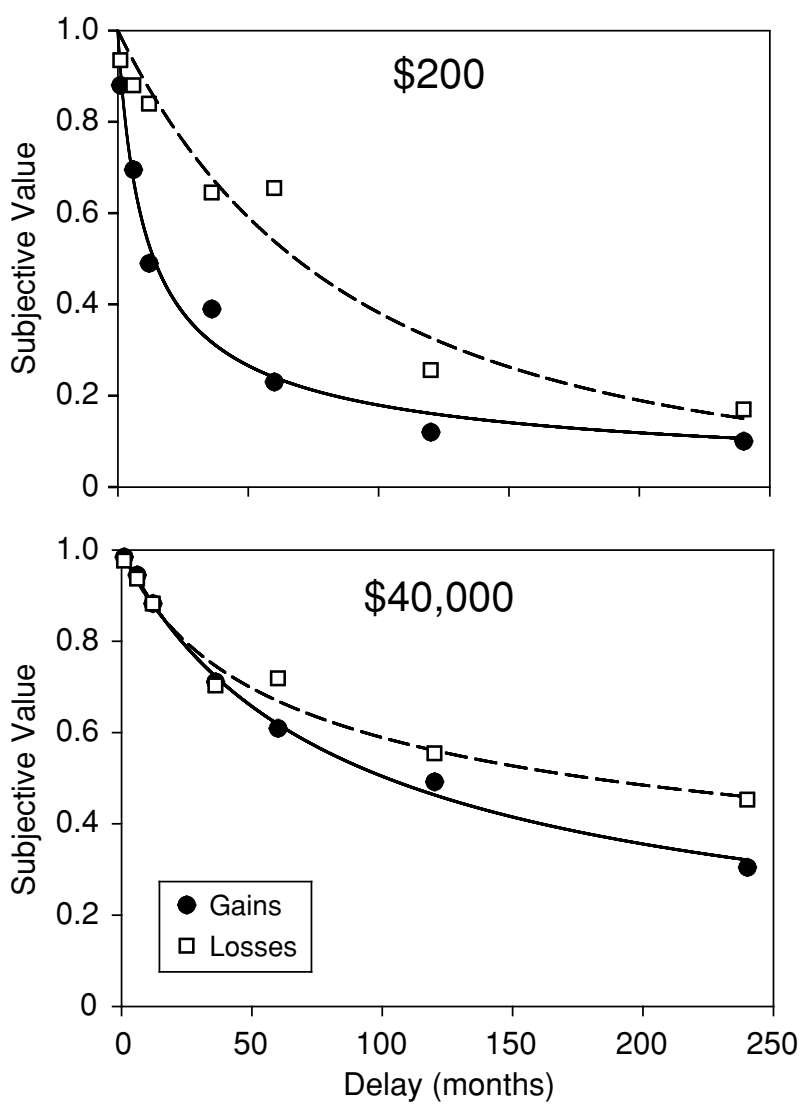

Figure 1. Subjective value as a function of the delay until a gain (filled circles) or loss (open squares) in Experiment 1. Symbols represent the group median subjective value expressed as a proportion of the $\$ 200$ (upper graph) and $\$ 40,000$ (lower graph) delayed outcomes. The curves represent Equation 1 fit to the data for gains (solid lines) or losses (dashed lines).

order to facilitate comparison of the discounting of gains and losses of different amounts, subjective value was calculated as a proportion of the actual delayed amount. (Note that although the subjective value of a loss is negative, when it is divided by the actual delayed amount, which is also negative, the resulting proportion is positive.) The curves represent Equation 1 fit to the group median data using a nonlinear, least squares algorithm. The results for the smaller $(\$ 200)$ and larger $(\$ 40,000)$ amounts are shown in the upper and lower graphs, respectively. The $R^{2} \mathrm{~S}$ for fits to the smaller and larger gains and the smaller and larger losses all were greater than .95 .

Equation 1 also was fit to individual data, resulting in four curves for each participant (one each for gains and losses at both the smaller and larger amounts, analogous to the four group curves shown in Figure 1). The pattern of individual results was similar to that observed at the group level. In general, Equation 1 provided good fits to the individual data, and the proportion of variance accounted for was higher for gains (median $R^{2} \mathrm{~S}$ of .95 for both $\$ 200$ 
and $\$ 40,000$ gains) than for losses (.91 and .84 for the $\$ 200$ and \$40,000 losses, respectively).

Typically, Equation 1 with $s<1.0$ provided a better fit to individual data than did a simple hyperbola (Equation 1 with $s=1.0$ ). The $s$ parameter was less than 1.0 in $72.5 \%$ of the cases for gains and $80.0 \%$ of the cases for losses. Moreover, the $s$ parameter was significantly less than 1.0 in $45.0 \%$ and $62.5 \%$ of the cases, respectively. In contrast, $s$ was never significantly greater than 1.0 . The fact that the temporal discounting function has two free parameters ( $b$ and $s$ ), however, creates problems for statistical comparisons between the degree of discounting in different conditions, as does the fact that distributions of these parameters are often highly skewed. Myerson, Green, and Warusawitharana (2001) proposed a way of dealing with these problems using an area-under-the-curve measure, in which the area is calculated on the basis of obtained subjective values (rather than on the basis of fitted discounting curves). This approach yields a single, approximately normally distributed, theoretically neutral measure of the degree of discounting. In calculating the area, subjective value is expressed as a proportion of the actual amount, and the independent variable (i.e., delay) is expressed as a proportion of the maximum value studied. Thus, area measures can range from 0 , indicating the steepest possible discounting, to 1.0, indicating no discounting.

Area measures were calculated for each participant for both gains and losses in each of the amount conditions (see Figure 2). These measures were then entered into a 2 (sign: gain vs. loss) $\times 2$ (amount: small vs. large) repeated measures ANOVA. The ANOVA revealed significant sign and amount (i.e., magnitude) effects. Specifically, gains were discounted more steeply than losses $[F(1,19)=16.07, p=.001]$, and small amounts were discounted more steeply than large amounts $[F(1,19)=6.93$, $p=.016]$, but the interaction failed to reach significance $[F(1,19)=3.65, p=.071]$.

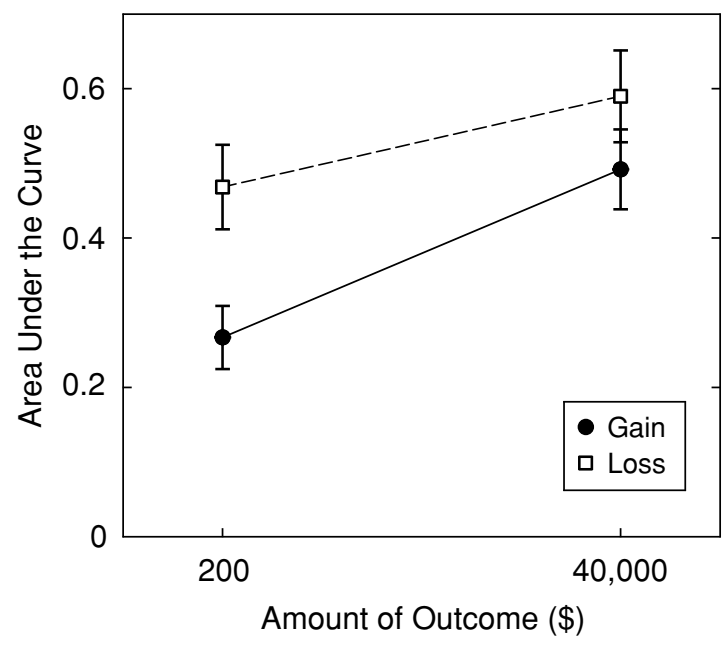

Figure 2. Area under the curve as a function of amount of delayed gain (filled circles) or delayed loss (open squares) in Experiment 1 . Error bars indicate \pm 1 standard error of the mean.

\section{Discussion}

With respect to the form of the discounting function, Equation 1 with $s$ less than 1.0 described well the relation between subjective value and delay at both the group and individual levels, regardless of whether the outcome involved gains or losses. Previous studies have found that a hyperbola provides a better fit for both gains and losses than does the exponential function predicted by standard microeconomic theory (e.g., Kirby \& Maraković, 1995; Murphy et al., 2001; Rachlin et al., 1991). However, Equation 1 with $s$ less than 1.0 provides even better fits to individual discounting data for gains than does a simple hyperbola, resulting in increases in explained variance that are significantly greater than would be expected just from adding a second parameter (e.g., Green et al., 1999; Myerson \& Green, 1995). The present results extend our previous findings with delayed gains to delayed losses. Estimates of the $s$ parameter for losses were typically less than 1.0, and in most cases significantly so. Moreover, $s$ values were never significantly greater than 1.0 for either gains or losses. These results strongly suggest that discounting of delayed outcomes, both gains and losses, is best described by a hyperbola-like function.

Comparison of the discounting functions for smaller and larger amounts revealed a magnitude effect for both delayed gains and delayed losses in which the smaller amount was discounted more steeply than the larger amount. With respect to gains, this result replicates a wellestablished finding (e.g., Green et al., 1997; Kirby, 1997). Although less is known regarding the effect of amount on the discounting of delayed losses, the present findings, showing that smaller losses are discounted more steeply than larger losses, are consistent with results reported by Thaler (1981) and Benzion et al. (1989).

With respect to the gain-loss asymmetry, the present results confirm the original observations by Thaler (1981) and Benzion et al. (1989) that delayed losses are discounted less steeply than delayed gains. This difference, known as the sign effect, is well established for probabilistic outcomes (e.g., Kahneman \& Tversky, 1979), but the difference between gains and losses has received much less attention with regard to delayed outcomes, and the present results strengthen the case for the generality of the sign effect.

Finally, note that although the amount $\times$ sign interaction was only at the trend level $(p=.07)$, the results shown in Figure 2 raise the possibility that the difference between gains and losses may be larger at smaller amounts. Consistent with this possibility, more participants showed steeper discounting for small versus large gains than for small versus large losses (90\% vs. 75\% of the participants). Moreover, among those showing steeper discounting of small than of large delayed outcomes, the magnitude effect (as measured by the ratio of the area for the large amount to the area for the small amount) was greater for delayed gains than for delayed losses $(2.57$ vs. 1.67). Because of this converging evidence consistent with the hypothesis that amount differentially affects the discounting of gains and losses, the issue of an amount $X$ 
sign interaction with delayed outcomes will be examined further in Experiment 3, after we consider a second experiment identical in design to Experiment 1 but involving probabilistic outcomes.

\section{EXPERIMENT 2}

In the second experiment, we addressed the same two basic questions regarding the discounting of gains and losses as we did in Experiment 1, but now directed at the discounting of probabilistic outcomes. The first of these questions concerned whether the mathematical function that best describes probability discounting of gains (i.e., Equation 1) also describes probability discounting when the outcomes are losses. As in Experiment 1, the effects of a smaller amount $(\$ 200)$ and a larger amount $(\$ 40,000)$ on discounting were compared in order to address the second question as to whether the magnitude effect in probability discounting of losses, if there is one, is similar to that observed in probability discounting of gains (for which large probabilistic losses are discounted more steeply than small losses).

\section{Method}

Participants. Twenty undergraduate students ( 9 males, 11 females; mean age $=19.3$ years) attending Washington University were recruited through the Department of Psychology's Human Subjects Pool and received course credit for their participation.

Procedure. The procedure was similar to that used in Experiment 1 and differed primarily in that the discounting task involved probabilistic rather than delayed outcomes. The gains phase and the losses phase each were divided into two blocks, one for the smaller amount and one for the larger amount. The order of the phases and the blocks was randomly determined.

For the gains phase of the probability discounting task, the participants first viewed on the computer monitor instructions similar to those for Experiment 1, except that the participants were told that when two amounts of money appeared on the screen, "One amount can be received FOR SURE. The other amount MIGHT POSSIBLY be received, and the screen will show you what the chances are that you will receive this amount. The FOR SURE amount of money will change after each of your decisions. The POSSIBLE amount of money will stay the same for a group of choices."

The gains phase consisted of two blocks of trials, one for each of the two certain ("for sure") amounts (\$200 and $\$ 40,000)$, in each of which the participant made six choices at each of seven probabilities $(.95, .90, .75, .50, .33, .10$, and .05$)$ presented in decreasing order. The iterative procedure for the gains phase of the probability discounting task was similar to that used in the gains phase of the temporal discounting task. The first choice at each probability was between an amount of money to be received "for sure" and half that amount to be received with a certain probability (e.g., $\$ 100$ for sure or $\$ 200$ with a $95 \%$ chance). For each of the subsequent trials, the amount of the certain gain was adjusted based on the participant's previous choice. If the participant chose the certain amount, then on the next trial this amount was decreased; if the participant chose the probabilistic amount, then the certain amount was increased on the next trial. As with the temporal discounting task, the size of the first adjustment was equal to half of the smaller amount, and the size of subsequent adjustments was always equal to half of the previous adjustment, rounded to the nearest dollar.

The subjective value of the probabilistic gain was calculated as being halfway between the largest certain amount that was preferred to the probabilistic amount and the smallest certain amount that was preferred to the probabilistic amount, except that if a participant always chose the probabilistic or the certain amount, then the subjective value was halfway between the last presented certain amount and the maximum (i.e., $\$ 200$ or $\$ 40,000$ ) or minimum (i.e., zero) amount, respectively.

The losses phase of the probability discounting task also consisted of two blocks of trials, one for each "for sure" amount, and began with instructions identical to those for the gains phase except that the word RECEIVE was replaced with the word PAY. The participants were asked to make a series of six choices at the same seven probabilities as in the gains phase, but choices were between an amount of money to be paid "for sure" and an amount of money to be paid with a stated probability, and the direction of the adjustments in the certain loss was opposite to that in the gains phase. That is, if the participant chose the certain loss, then on the next trial the amount of that alternative was increased, and if the participant chose the probabilistic loss, then the certain amount was decreased on the next trial. The subjective value of the probabilistic loss was calculated in a manner similar to that used to calculate the subjective value of the probabilistic gain.

\section{Results}

Figure 3 presents the group median subjective value (expressed as a proportion of the actual amount of the probabilistic outcome so as to facilitate comparisons be-
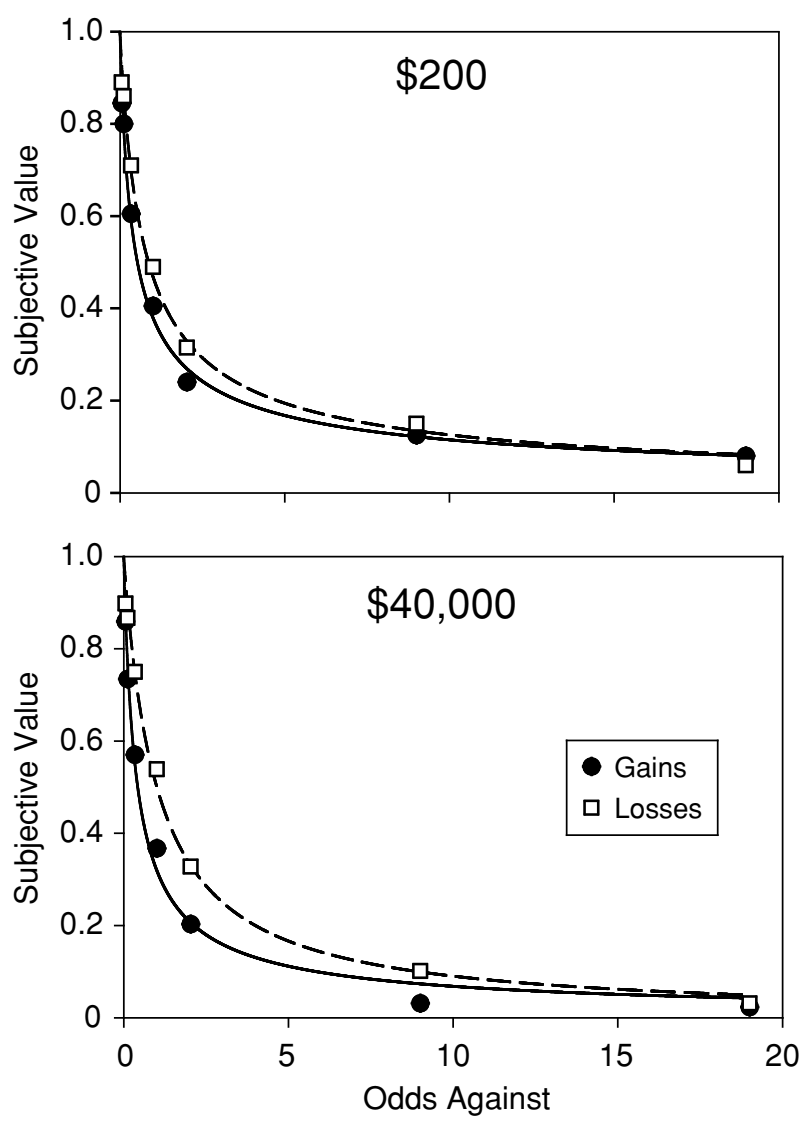

Figure 3. Subjective value as a function of the odds against a gain (filled circles) or loss (open squares) in Experiment 2. Symbols represent the group median subjective value expressed as a proportion of the $\$ 200$ (upper graph) and $\$ 40,000$ (lower graph) probabilistic outcomes. The curves represent Equation 1 fit to the data for gains (solid lines) or losses (dashed lines). 
tween the different amounts) plotted as a function of the odds against receiving or paying the specified amount. The curves represent Equation 1 fit to the group median data. The $R^{2} \mathrm{~s}$ for fits to the smaller (upper graph) and larger (lower graph) gains and losses all were greater than .98 .

Equation 1 also was fit to each individual's probability discounting data and typically provided good fits to the data (median $R^{2}$ s greater than .95 for both smaller and larger gains and for both smaller and larger losses). The $s$ parameter was less than 1.0 in $75.0 \%$ of the cases for gains and in $57.5 \%$ of the cases for losses. Moreover, the $s$ parameter was significantly less than 1.0 in $47.5 \%$ and $30.0 \%$ of the cases, respectively, whereas $s$ was never significantly greater than 1.0 .

Area measures of discounting were calculated separately for each participant for both gains and losses at both amounts (see Figure 4), and a $2 \times 2(\operatorname{sign} \times$ amount of outcome) repeated measures ANOVA was conducted. (In examining Figure 4, recall that the area measure can range from 0 to 1.0 , with lower values representing steeper discounting.) The ANOVA revealed a main effect of amount, with the larger amount being discounted more steeply than the smaller amount $[F(1,19)=13.96, p=.001]$, but the difference between gains and losses failed to reach significance $[F(1,19)=3.32, p=.084]$. There was no interaction $[F(1,19)<1.0]$.

\section{Discussion}

The results of the second experiment extend the findings of Experiment 1 in showing that a hyperbola-like function (Equation 1) describes both the discounting of probabilistic gains and the discounting of probabilistic losses. Although it has been shown previously that Equation 1 accurately describes choices involving probabilistic gains, and does so better than a simple hyperbola, the present results are the first to extend this finding to choices

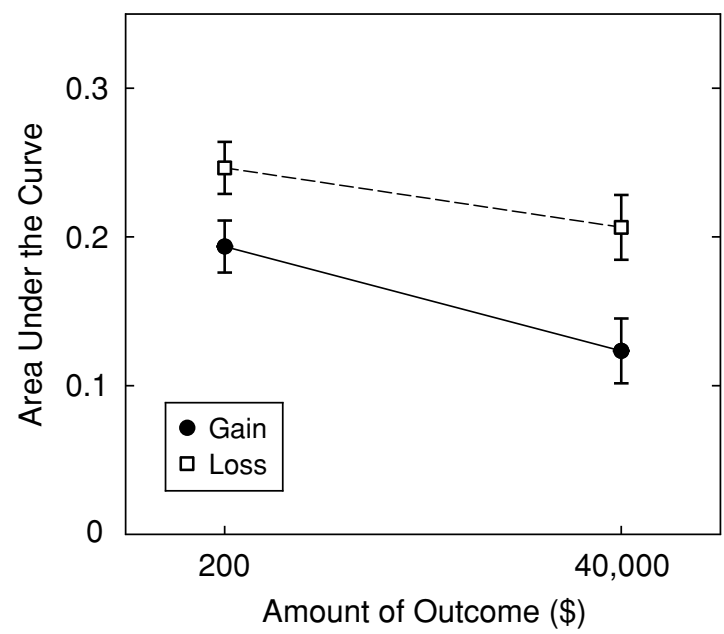

Figure 4.Area under the curve as a function of amount of probabilistic gain (filled circles) or probabilistic loss (open squares) in Experiment 2. Error bars indicate \pm 1 standard error of the mean. involving probabilistic losses. For both gains and losses, estimates of the $s$ parameter were often significantly less than 1.0, and never significantly greater than 1.0.

The present results are similar to those of Tversky and Kahneman (1992), who showed that a single model, cumulative prospect theory, could describe choices involving probabilistic gains as well as choices involving probabilistic losses. There are two important differences, however, between our approach and that of Tversky and Kahneman. First, whereas cumulative prospect theory applies specifically to probabilistic outcomes, the hyperbola-like model may be applied to both delayed and probabilistic outcomes. Second, Tversky and Kahneman's model requires fitting both a value function (relating subjective value to amount) and a weighting function (relating probability to the weight given a choice alternative), rather than fitting a single (hyperbola-like) probability discounting function. Although the hyperbola-like discounting function also may be decomposed into value and weighting functions (Green \& Myerson, 2004), no attempt to do so was undertaken here. Rather, our focus was on comparing the discounting of gains and losses as well as the discounting of delayed and probabilistic outcomes using the same experimental procedures and analytic techniques.

Comparison of the probability discounting functions for smaller and larger amounts revealed a magnitude effect for both gains and losses that was opposite in direction to the magnitude effect observed with delayed outcomes. For both probabilistic gains and losses, the smaller amount was discounted less steeply than the larger amount. With respect to gains, the magnitude effect observed in the present experiment replicates previous results (e.g., Green et al., 1999; Myerson et al., 2003), and the present results extend this finding to probabilistic losses.

Although the amount $\times$ sign interaction was not significant, more participants showed steeper discounting for large versus small gains than for large versus small losses ( $75 \%$ vs. $60 \%$ of the participants). Moreover, among those showing shallower discounting of small than of large probabilistic outcomes, the magnitude effect (as measured by the ratio of the area for the small amount to the area for the large amount) was greater for probabilistic gains than for probabilistic losses $(2.22$ vs. 1.50$)$. (Note that the ratio used here to measure the magnitude effect for probabilistic outcomes is the inverse of that for delayed outcomes because the two magnitude effects are in opposite directions.) These results obtained with probabilistic outcomes, like those obtained with delayed outcomes in Experiment 1, suggest that magnitude effects for losses tend to be smaller and less reliable than those for gains.

As Green and Myerson (2004) pointed out, magnitude effects pose problems for current models of discounting. Neither prospect theory (Kahneman \& Tversky, 1979; Tversky \& Kahneman, 1992) nor the present model (Equation 1) predicts the effect of amount on probability discounting, although both can account for the form of the discounting function and can accommodate magnitude effects when they occur. In contrast, Prelec and Loewenstein (1991) have proposed a model that does predict a 
magnitude effect, but the predicted effect is opposite in direction to that observed. Because of its potential theoretical significance, the issue of the effect of amount on the discounting of probabilistic outcomes is examined in greater detail in Experiment 4.

With respect to the gain-loss asymmetry, prospect theory (Kahneman \& Tversky, 1979; Tversky \& Kahneman, 1981) predicts greater discounting of gains than of losses because the value function for losses is hypothesized to be steeper than that for gains. In the present experiment, the difference between area measures of discounting gains and losses was only at the trend level, although it was in the predicted direction, and this issue is also reexamined in Experiment 4.

\section{EXPERIMENT 3}

The results of our first experiment raised the possibility that gains and losses differ in the extent to which the amount of a delayed outcome affects how steeply it will be discounted. That is, the difference between the extent to which delayed gains and losses were discounted in Experiment 1 appeared to be greater for smaller than for larger amounts (see Figure 2). Although the interaction was only at the trend level, the effect of amount was both more reliable (i.e., seen in a higher percentage of participants) and stronger when the outcomes were gains than when the outcomes were losses. Accordingly, in Experiment 3 we varied the amounts over a greater range in order to better assess the possibly differential effects of amount on the discounting of delayed gains and losses. Specifically, we compared the discounting of gains and losses of $\$ 100$, $\$ 20,000$, and $\$ 60,000$. At issue was whether the size of the gain-loss asymmetry (i.e., greater discounting of gains than losses) would vary as a function of the amount of the delayed outcome.

\section{Method}

Participants. Twenty-seven undergraduate students (15 males, 12 females, mean age $=19.2$ years) attending Washington University were recruited through the Department of Psychology's Human Subjects Pool. All the participants received course credit for their participation and had not participated in either of the two previous experiments.

Procedure. The procedure for Experiment 3 was identical to that for Experiment 1 except for the delays and the amounts studied. The gains and losses phases of the temporal discounting task each consisted of three amount conditions $(\$ 100, \$ 20,000$, and $\$ 60,000)$. In each of these conditions, the participants made six choices at each of eight delays ( 1 month, 6 months, 1 year, 3 years, 5 years, 8 years, 12 years, and 20 years).

\section{Results}

Figure 5 shows the group median subjective value of the delayed outcome, expressed as a proportion of the actual amount, plotted as a function of the time until receipt or payment. The curves represent Equation 1 fit to the data and accounted for more than $96 \%$ of the variance in each of the six cases depicted.

Equation 1 also was fit to the data for each individual. For gains, the median $R^{2}$ s were $.94, .90$, and .95 , for the
$\$ 100, \$ 20,000$, and $\$ 60,000$ amounts, respectively. For losses, the corresponding median $R^{2} \mathrm{~s}$ were $.74, .85$, and .82. The $s$ parameter was less than 1.0 in $69.1 \%$ of the cases for gains and in $74.1 \%$ of the cases for losses. Moreover, the $s$ parameter was significantly less than 1.0 in $49.4 \%$ and $61.7 \%$ of the cases for gains and losses, respectively, and never significantly greater than 1.0.

Area measures of discounting were calculated for each participant for both gains and losses in each of the amount conditions (see Figure 6). Tests of within-subjects contrasts revealed significant linear and quadratic trends in the effects of amount on the degree of discounting $[F(1,26)=$ $31.385, p<.001$, and $F(1,26)=25.71, p<.001]$, as well as a significant sign effect $[F(1,26)=10.18, p=.004]$,
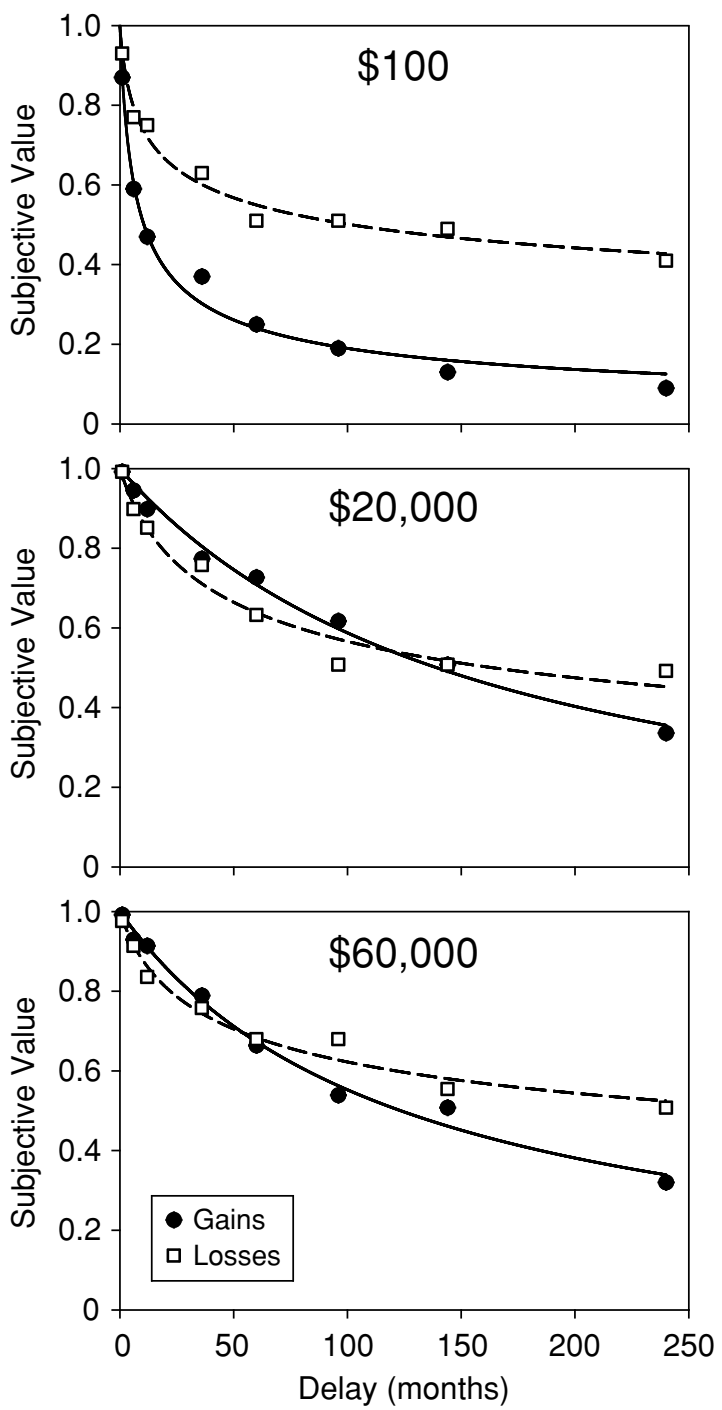

Figure 5. Subjective value as a function of the delay until a gain (filled circles) or loss (open squares) in Experiment 3. Symbols represent the group median subjective value expressed as a proportion of the $\$ 100$ (upper graph), $\$ 20,000$ (middle graph), and $\$ 60,000$ (lower graph) delayed outcomes. The curves represent Equation 1 fit to the data for gains (solid lines) or losses (dashed lines). 


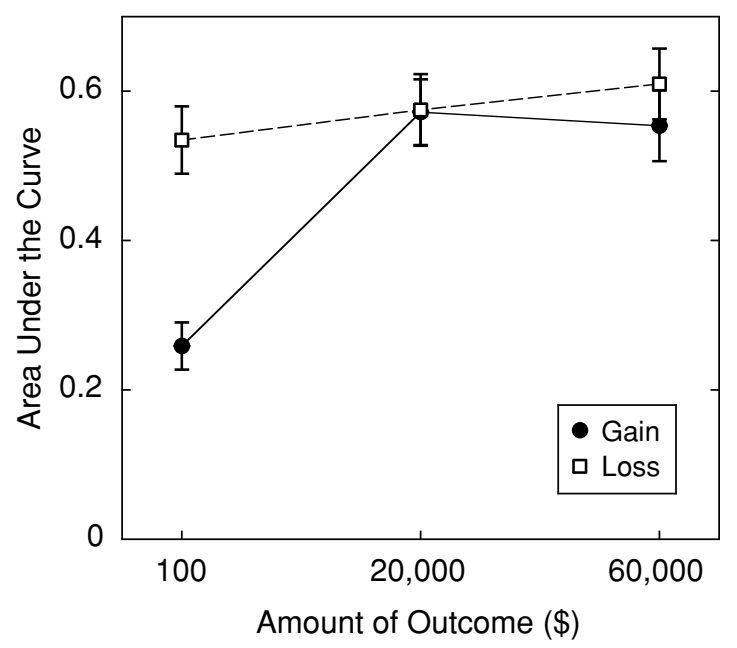

Figure 6. Area under the curve as a function of amount of delayed gain (filled circles) or delayed loss (open squares) in Experiment 3. Error bars indicate \pm 1 standard error of the mean.

reflecting the fact that gains were discounted more steeply than losses (as indicated by smaller areas). In addition, there were significant linear and quadratic sign $\times$ amount interactions $[F(1,26)=14.74, p=.001$, and $F(1,26)=$ $16.44, p<.001$, respectively].

To explicate these interactions, we conducted separate one-way ANOVAs on the data from the gains and losses conditions. For gains, there were significant linear and quadratic trends $[F(1,26)=54.11, p<.001$, and $F(1,26)=48.88, p<.001]$ resulting from the fact that the small delayed amount was discounted more steeply than the two larger delayed amounts, which were discounted to a similar degree. For losses, neither the linear nor the quadratic trend was significant $[F(1,26)=2.56, p=$ .122 ; and $F(1,26)<1.0]$. Thus, the interactions between amount and sign of outcome that may be seen in Figure 6 reflect the fact that the discounting of delayed gains was significantly affected by amount, whereas the discounting of delayed losses was not. A parallel finding is that with delayed gains, $89 \%$ of the participants showed steeper discounting of the $\$ 100$ outcome than of the $\$ 60,000$ outcome, whereas only $70 \%$ showed this pattern with delayed losses. Moreover, among those showing such magnitude effects, the average effect size (as measured by the ratio of the area for the larger amount to the area for the smaller amount) was 3.60 for gains but only 1.61 for losses.

\section{Discussion}

Delayed gains were discounted more steeply than delayed losses of the same amount in Experiment 3, and small delayed gains were discounted more steeply than larger delayed gains. These findings replicate and extend the findings of the first experiment. Recall, however, that in Experiment 1 the effect of amount on the discounting of delayed losses appeared to be less than the effect of amount on the discounting of delayed gains, although the interaction between sign and amount did not quite reach statistical significance. In the present experiment, we examined a larger range of amounts than were examined in either Experiment 1 or in previous studies (Benzion et al., 1989; Thaler, 1981; Yates \& Watts, 1975), and a significant interaction between these factors was observed. Across this range, the discounting of delayed gains decreased nonlinearly as amount was increased. In contrast, the discounting of delayed losses was relatively unaffected by amount, leading to a greater gain-loss asymmetry when the delayed amounts were small than when the delayed amounts were large. As far as we know, these results represent the first reported instance of this potentially theoretically important interaction.

\section{EXPERIMENT 4}

The results of the preceding experiment on discounting of delayed gains and losses demonstrate the utility of examining the effects of amount over a large range. Accordingly, Experiment 4 examined the effect of amount on the discounting of probabilistic gains and losses over the same range of amounts as that used in Experiment 3. Magnitude effects are of considerable theoretical interest because, contrary to the predictions of theories that assume that the same or similar processes underlie both types of discounting (e.g., Prelec \& Loewenstein, 1991; Rachlin et al., 1986), previous studies have reported that amount of outcome affects temporal and probability discounting of gains in opposite directions. Given the significance of the findings regarding gains, the effect of amount on the discounting of losses bears careful examination. To date, however, this topic has received little attention in the experimental literature. In addition, the interaction between sign and amount observed with delayed outcomes in Experiment 3 raises the question of whether an analogous interaction would be observed with probabilistic outcomes if a corresponding range of amounts were studied, and therefore Experiment 4 revisited this issue.

\section{Method}

Participants. Twenty-six undergraduate students (15 males, 11 females, mean age $=19.8$ years) attending Washington University were recruited through the Department of Psychology's Human Subjects Pool. All the participants received course credit for their participation and had not participated in any of the previous three experiments.

Procedure. The procedure for Experiment 4 was identical to that for Experiment 2 except for changes in the probabilities and amounts studied. Both the gains and losses phases of the probability discounting task consisted of three amount conditions $(\$ 100$, $\$ 20,000$, and $\$ 60,000)$. In each of these conditions, the participants made their series of choices at each of eight probabilities $(.95, .90$, $.75, .50, .33, .20, .10$, and .05).

\section{Results}

Figure 7 shows the group median subjective value of the probabilistic outcome, expressed as a proportion of the actual amount, plotted as a function of the odds against receiving the reward or having to make the payment. The curves represent Equation 1 fit to the data and accounted for more than $98 \%$ of the variance in each of the six cases shown. 

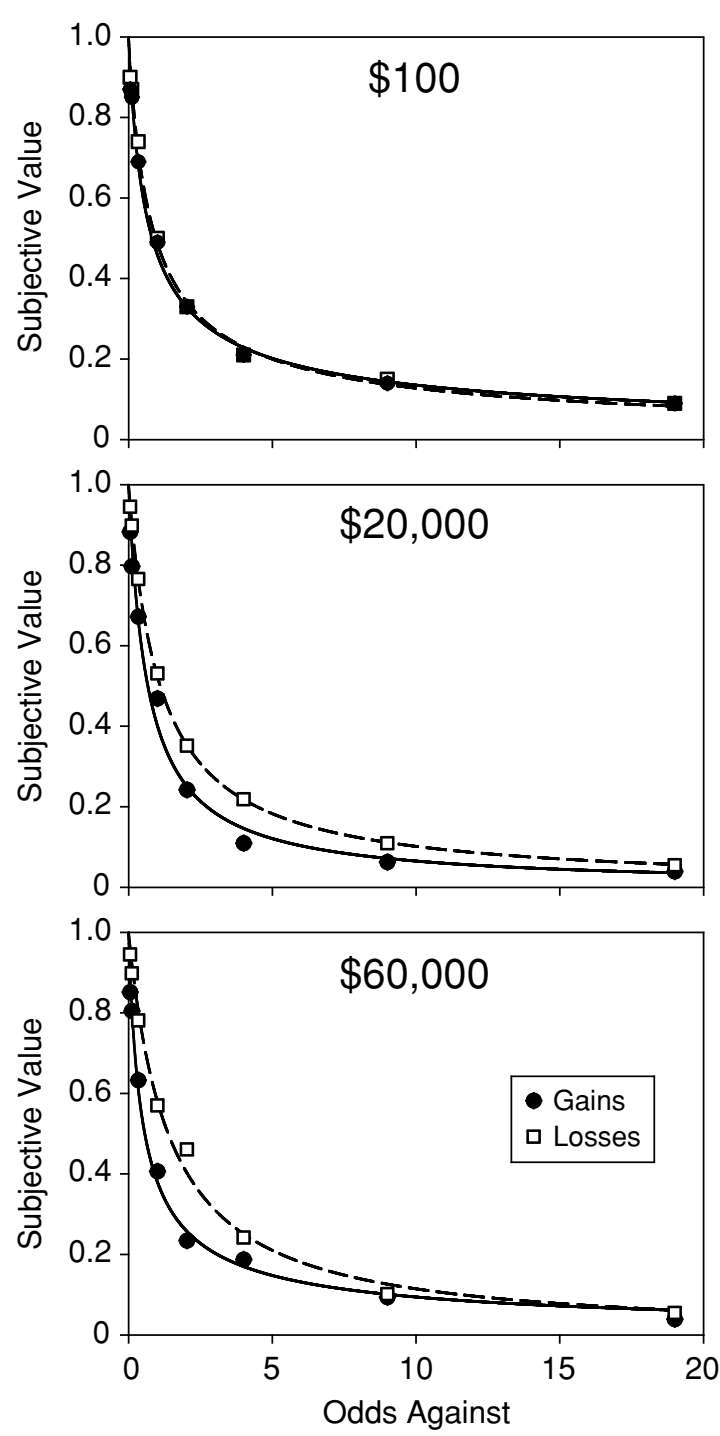

Figure 7. Subjective value as a function of the odds against a gain (filled circles) or loss (open squares) in Experiment 4. Symbols represent the group median subjective value expressed as a proportion of the $\$ 100$ (upper graph), $\$ 20,000$ (middle graph), and $\$ 60,000$ (lower graph) probabilistic outcomes. The curves represent Equation 1 fit to the data for gains (solid lines) or losses (dashed lines).

Equation 1 also was fit to the data for each individual. For gains, the median $R^{2} \mathrm{~s}$ were $.98, .97$, and .98 , for the $\$ 100, \$ 20,000$, and $\$ 60,000$ amounts, respectively. For losses, the corresponding median $R^{2} \mathrm{~s}$ were $.95, .96$, and .95 . The $s$ parameter was less than 1.0 in $64.1 \%$ of the cases for gains and $65.4 \%$ of the cases for losses. Moreover, the $s$ parameter was significantly less than 1.0 in $34.6 \%$ of the cases for gains as well as for losses, and was never significantly greater than 1.0 .

Area measures of discounting were calculated for each participant for both gains and losses in each of the amount conditions (see Figure 8). Tests of within-subjects con- trasts revealed a significant sign effect $[F(1,25)=7.35$, $p=.012]$, reflecting the fact that gains were discounted more steeply than losses (as indicated by the smaller area values for gains), but there were no linear or quadratic trends in the effect of amount $[F(1,25)=2.70, p=.113$, and $F(1,26)<1.0$, respectively]. However, both the linear and the quadratic interactions between sign and amount were statistically significant $[F(1,25)=9.92, p=.004$, and $F(1,25)=12.20, p=.002$, respectively].

To explicate these interactions, we conducted separate one-way ANOVAs on the gains and losses data. For gains, there were significant linear and quadratic trends $[F(1,25)=8.59, p=.007$, and $F(1,25)=19.69, p<.001]$, resulting from the fact that the small probabilistic amount was discounted less steeply than the two larger probabilistic amounts, which were discounted to a similar degree. For losses, neither the linear nor the quadratic trend was significant $[F(1,25)=1.83, p=.189$, and $F(1,25)=1.12$, $p=.301]$. Thus, the interactions between amount and sign of outcome that may be seen in Figure 8 reflect the fact that the discounting of probabilistic losses was not significantly affected by amount, whereas the discounting of probabilistic gains was affected. Consistent with this differential effect of amount, $77 \%$ of the participants discounted the $\$ 100$ outcome more steeply than they did the $\$ 60,000$ outcome when the outcomes were gains, whereas less than half (35\%) showed this pattern when the outcomes were losses. Moreover, among those showing such magnitude effects, the average effect size (as measured by the ratio of the area for the smaller amount to the area for the larger amount) was 1.99 for gains but only 1.20 for losses.

\section{Discussion}

The results of Experiment 4 revealed that probabilistic gains were discounted more steeply than losses and that

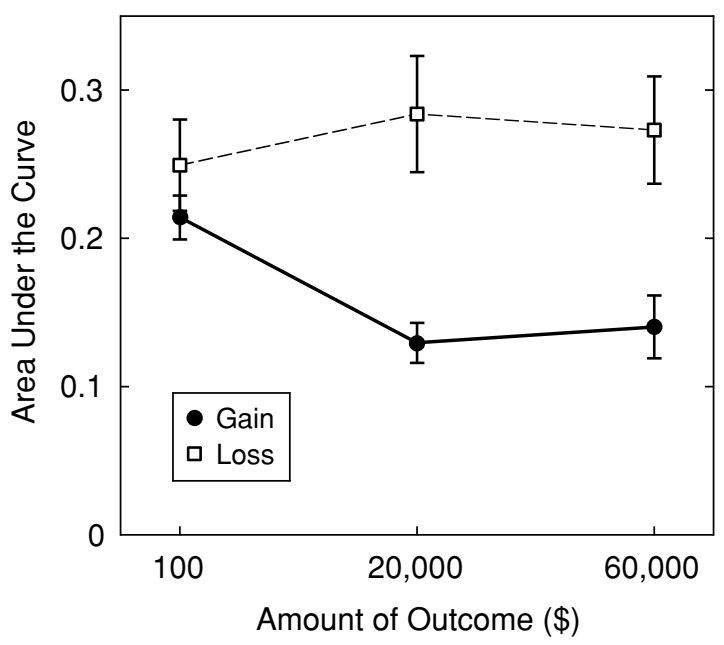

Figure 8. Area under the curve as a function of amount of probabilistic gain (filled circles) or probabilistic loss (open squares) in Experiment 4. Error bars indicate \pm 1 standard error of the mean. 
small probabilistic gains were discounted less steeply than larger probabilistic gains. Importantly, there was a significant interaction between amount and sign of outcome, reflecting the fact that the discounting of probabilistic gains increased nonlinearly as amount was increased, whereas the discounting of probabilistic losses was relatively unaffected by amount. This interaction, in turn, resulted in a greater gain-loss asymmetry when the probabilistic amounts were large than when they were small. On the one hand, this finding is the reverse of that observed in the previous experiment on delayed outcomes, in which a greater gain-loss asymmetry was found for small amounts than for large amounts. On the other hand, the present results are similar to the results of the previous experiment in that both experiments found weaker and less reliable effects of amount on the degree to which losses were discounted, compared with gains, regardless of whether the outcomes were delayed (as in Experiment 3) or probabilistic (as in Experiment 4).

\section{GENERAL DISCUSSION}

The present study is the first to compare and contrast temporal and probability discounting of gains and losses across a broad range of amount conditions ( $\$ 100$ to $\$ 60,000)$ using similar experimental procedures and analytical techniques, and also the first to address the issue of whether the same mathematical function can describe the discounting of delayed gains, delayed losses, probabilistic gains, and probabilistic losses. Taken together, the results of all four experiments revealed that the gain-loss asymmetry decreases with the amount of delayed outcome, but increases with the amount of probabilistic outcome. With regard to the form of the discounting function, the results show that discounting functions with the same hyperbolalike mathematical form, Equation 1, can describe both temporal and probability discounting, regardless of whether the outcomes are gains or losses.

For delayed and probabilistic gains, these findings regarding the form of the discounting function replicate the results of previous studies demonstrating the superiority of the hyperbola-like function (for a review, see Green \& Myerson, 2004). There has been little research, however, on the form of the discounting function for losses, either delayed or probabilistic. Although Murphy et al. (2001) reported that a simple hyperbola provided better fits than did an exponential decay function to both delayed gains and losses, they only examined one amount and did not evaluate Equation 1. Further analyses of Murphy et al.'s data by Green and Myerson (2004) revealed that for both gains and losses, Equation 1 with an exponent less than 1.0 provided significantly better fits than did a hyperbola (i.e., Equation 1 with the exponent equal to 1.0). The present results extend this finding to a broad range of amounts and also to probabilistic outcomes.

The general applicability of the hyperbola-like discounting function, by itself, might be taken as support for the hypothesis that similar processes underlie the discounting of both delayed and probabilistic outcomes. The finding that amount has different effects on temporal and probability discounting, however, argues against such an interpretation. First, as amount increases, delayed gains are discounted less steeply, whereas probabilistic gains are discounted more steeply. This result, observed in the present study as well as in previous studies (e.g., Du et al., 2002; Green et al., 1999), violates the predictions of single-process theories. That is, if the same process were involved in both kinds of discounting, then one would expect that the same manipulation (e.g., increases in the amount of reward) would produce the same effect on both temporal and probability discounting, whereas opposite effects are observed. Interestingly, amount had smaller and less reliable effects on the discounting of losses, whether delayed or probabilistic.

Second, the amount of probabilistic reward recently has been reported to affect the exponent of individuals' discounting functions, whereas the amount of delayed reward does not affect the exponent (Myerson et al., 2003). This finding represents yet another difference between temporal and probability discounting and thus creates further problems for accounts that assume that the same or similar principles are involved in both (e.g., Prelec \& Loewenstein, 1991; Rachlin et al., 1986). Moreover, although Myerson et al.'s (2003) results are consistent with the derivation of the temporal discounting function proposed by Myerson and Green (1995), they are inconsistent with an analogous derivation of the probability discounting function (Green \& Myerson, 2004).

In both cases, the derivations are based on psychophysical scaling of the relevant dimensions (time and money for temporal discounting and odds and money for probability discounting), and they imply that within a specific domain (i.e., either temporal or probability discounting), the exponent should remain constant across different choice situations even if different amounts of money are involved. The data from the present experiments replicate the previous findings for delayed and probabilistic gains (Myerson et al., 2003) and, importantly, extend them to losses. As may be seen in Figure 9, which plots the present data (circles and squares) along with those from Myerson et al. (triangles), for both delayed gains and losses the median values of the $s$ parameter did not change systematically as a function of the amount of the outcome (upper graph). In contrast, the median values of the $s$ parameter increased with the amount of probabilistic outcome (lower graph). Thus, although the present results strongly support a derivation of the temporal discounting function based on psychophysical scaling, they equally clearly fail to support an analogous derivation of the probability discounting function, again calling attention to the difference between choice involving delayed outcomes and choice involving probabilistic outcomes.

A major finding of the present study is that the amount of loss has relatively little effect on the extent to which either delayed or probabilistic losses are discounted. This finding was unexpected in light of the robustness of the 

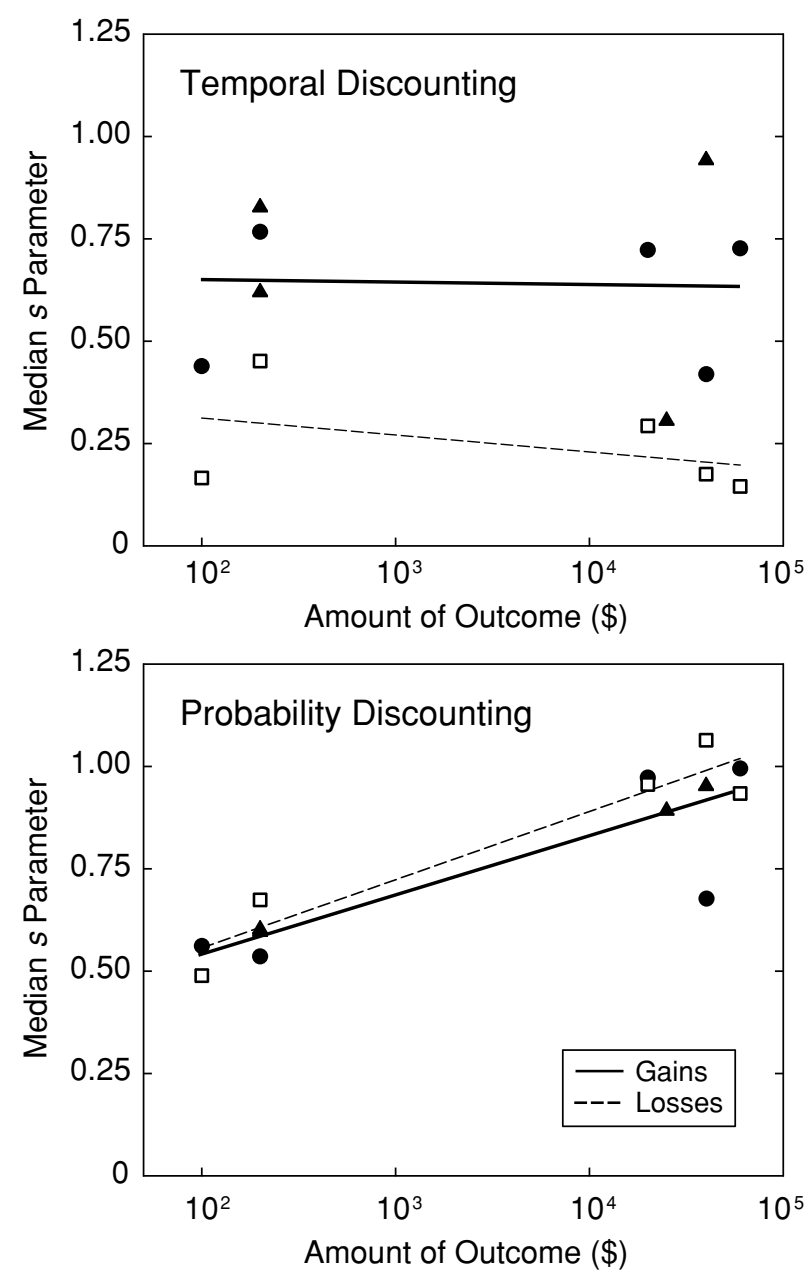

Figure 9. Median $s$ parameter as a function of amount of delayed (upper graph) or probabilistic (lower graph) gain (solid symbols) or loss (open symbols). The circles and squares represent data from Experiments 1-4 of the present study, and the triangles represent data from Myerson et al. (2003). The solid and dashed lines represent the regression lines for gains and losses, respectively. Note that amount is logarithmically scaled.

magnitude effects observed with delayed and probabilistic gains. Even in the case of delayed gains, however, where steeper discounting of smaller amounts has been observed repeatedly (e.g., Chapman \& Elstein, 1995; Green et al., 1997; Kirby, 1997; Raineri \& Rachlin, 1993), the source of the magnitude effect is unclear, although numerous conjectures have been offered.

Loewenstein and Prelec (1992), for example, proposed a model of choice with delayed outcomes in which, as in prospect theory (Kahneman \& Tversky, 1979), the discounting function is decomposed into a value function and a weighting function. Loewenstein and Prelec suggested that the shape of the value function is subproportional, and that as a result, smaller delayed gains are discounted more steeply than larger delayed gains. Raineri and Rachlin (1993) offered a similar explanation for the magnitude effect. They proposed that individuals do not choose between amounts of gain, per se, but between consumption rates, and also that the relationship between consumption rate and amount is subproportional. These assumptions would explain the observed magnitude effect for delayed gains. Myerson and Green (1995) discussed two possible explanations for the magnitude effect, one based on differences in the frequency of choice opportunities involving smaller and larger amounts and the other based on differences in the hazard rates associated with waiting for smaller and larger amounts. Loewenstein and Thaler (1989) also considered two possibilities, one based on the idea that perception incorporates absolute as well as relative differences between amounts and the other based on the idea that people maintain separate mental accounts for small gains (windfalls) and large gains (savings).

All of the preceding conjectures run into problems, however, because, as observed in the present study, large probabilistic gains are discounted more steeply than small probabilistic gains, whereas the opposite pattern is observed with delayed gains. For example, Prelec and Loewenstein (1991) noted that their model localizes magnitude effects in the shape of the value function, and as a result, incorrectly predicts similar effects of amount for both delayed and probabilistic gains. Each of the other conjectures also fails to predict the opposite effects of amount on temporal and probability discounting.

To deal with this problem, Prelec and Loewenstein (1991) invoked the greater disappointment experienced when a gamble involving large stakes is lost to explain the fact that large probabilistic gains are discounted more steeply than small probabilistic gains. Thus, their account builds on the fact that although choices between an immediate and a delayed gain involve only the two outcomes, choices between a certain and a probabilistic gain involve three outcomes: the certain gain, the probabilistic gain, and the possibility of no gain. In the latter case, they assumed that people experience disappointment when they lose the gamble and receive nothing. Green et al. (1999) made a similar suggestion, although they did not invoke disappointment but simply treated the possibility of not winning the gamble as a loss. Again, the higher the stakes, the greater the potential loss would be.

Thus, both Prelec and Loewenstein's (1991) and Green et al.'s (1999) accounts assume, first, that choices involving probabilistic gains implicitly involve possible losses which are counted against the possible gains, and second, that as the amounts involved are increased, the negative subjective value of the possible loss increases more than the positive subjective value of the gain. This hypothesized greater effect of amount on the subjective value of losses leads to magnitude effects on probabilistic gains that are opposite in direction to those observed when just gains are involved (as in the case of delayed rewards). As the present study shows, however, amount has relatively little effect on the discounting of losses, and thus both Prelec and Loewenstein's (1991) and Green et al.'s (1999) conjectures regarding the source of magnitude effects in temporal and probability discounting are called into question. Future theoretical work will need to take into account the fact that not only does amount have different effects 
on discounting depending on whether the outcomes are delayed or probabilistic, but also amount has different effects depending on whether the outcomes are gains or losses.

Although we do not yet know the correct explanation of the magnitude effects observed with delayed and probabilistic gains, on the basis of the present findings we now know that the robust magnitude effects for gains in combination with the much smaller and less reliable magnitude effects for losses result in amount-dependent changes in the gain-loss asymmetry. As may be seen in Figure 10, which combines data from all four experiments, amount had a much smaller effect on the discounting of delayed losses than on the discounting of delayed gains (upper graph), and had little or no effect on the discounting of probabilistic losses (lower graph). As a result, the difference between discounting of delayed gains and losses decreased as a function of amount, whereas the difference between discounting of probabilistic gains and losses increased as a function of amount.

In a study examining temporal discounting of hypothetical gains and losses in the health and monetary domains, Chapman (1996) failed to observe an interaction between sign and amount, even though main effects of both amount and sign were observed on both health and monetary outcomes. In contrast, Baker et al. (2003) compared temporal discounting by cigarette smokers and nonsmokers, and observed results similar to those in the present study. For both smokers and nonsmokers, the discounting of delayed gains was more affected by amount than was the discounting of delayed losses, so that, as in the present study, the difference between discounting rates for gains and losses was smaller at higher amounts. Moreover, the interaction between the sign (gain vs. loss) and amount of the delayed outcome was observed with both hypothetical monetary and hypothetical cigarette outcomes. The present study extends these findings to probability discounting of gains and losses and, importantly, demonstrates that although the gain-loss asymmetry is observed with both delayed and probabilistic outcomes, the asymmetry decreases with the amount of the delayed outcome but increases with the amount of the probabilistic outcome.

In summary, even though the same hyperbola-like function describes the temporal and probability discounting of both gains and losses, important differences are observed in the effects of amount on discounting. These differential effects represent an interesting pattern of similarities and differences that, in combination, produce opposite patterns when one compares the extent to which delayed and probabilistic outcomes are discounted. That is, unlike the discounting of gains, the extent to which losses are discounted is relatively insensitive to the amounts involved, and this is true regardless of whether losses are delayed or probabilistic. This insensitivity, in combination with the opposite effects that amount has on temporal and probability discounting of gains, gives rise to changes in gainloss asymmetry that go in opposite directions, decreasing with amount for delayed outcomes and increasing with
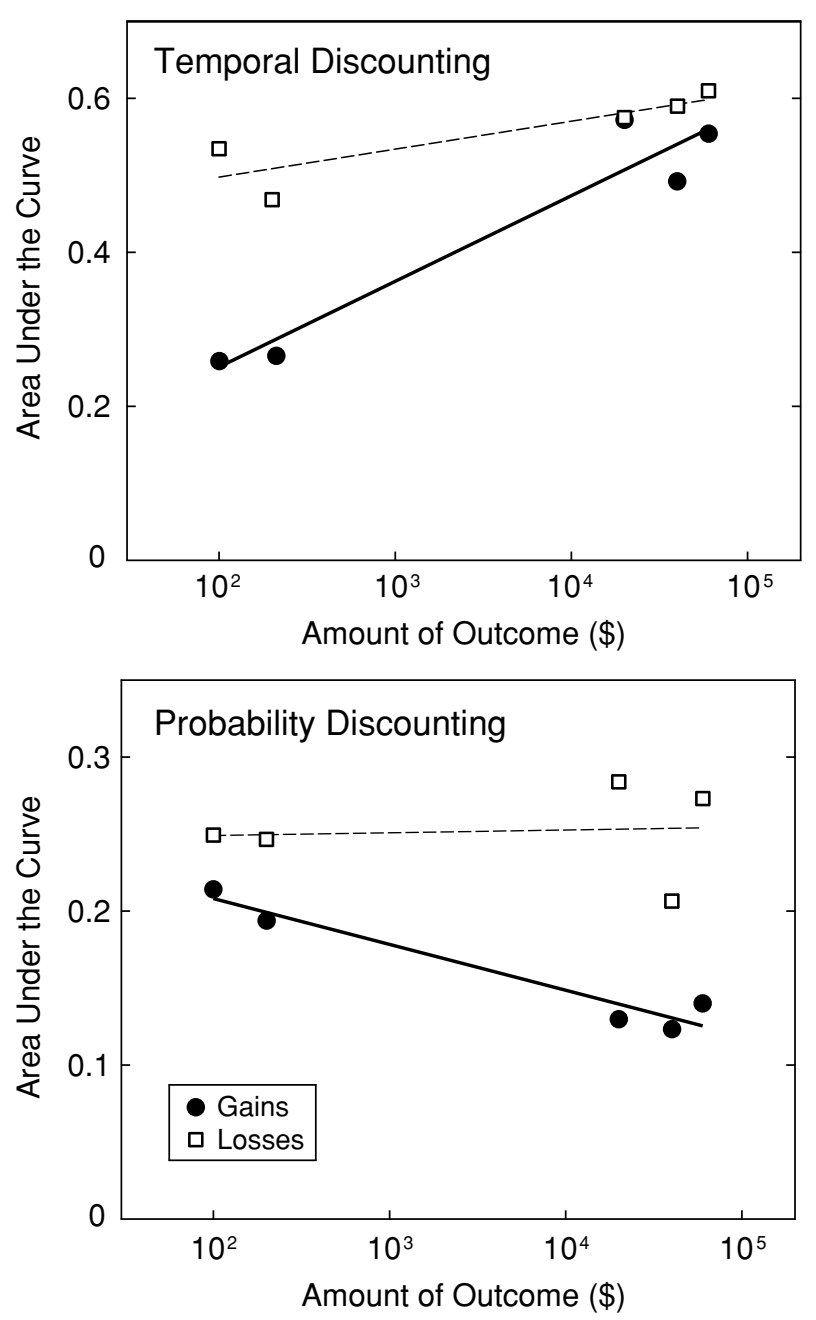

Figure 10. Area under the curve as a function of amount of delayed (upper graph) or probabilistic (lower graph) gain (filled circles) or loss (open squares). Data shown are from all four experiments. The solid and dashed lines represent the regression lines for gains and losses, respectively. Note that amount is logarithmically scaled.

amount for probabilistic outcomes. The fact that amount differentially affects the discounting of gains and losses suggests that different decision-making processes are involved in these two domains, just as the different effects of amount on delayed and probabilistic gains argue that different processes are involved in temporal and probability discounting.

The obtained pattern of results places important constraints on theorizing about these different processes. For example, the robust magnitude effect observed with gains has been explained by positing separate mental accounts for small and large amounts (e.g., Loewenstein \& Thaler, 1989). It is not immediately apparent, however, how the hypothesis of separate accounts can be reconciled with the observed relative insensitivity to amount of loss. In brief, although the failure of current general theories of 
discounting would seem to indicate a need for more specific explanations, such explanations cannot be developed without confronting the rich overall pattern of results that is revealed when delayed and probabilistic gains and losses are considered simultaneously.

\section{REFERENCES}

AINSLIE, G. (1992). Picoeconomics: The strategic interaction of successive motivational states within the person. Cambridge: Cambridge University Press.

BAKer, F., Johnson, M. W., \& Bickel, W. K. (2003). Delay discounting in current and never-before cigarette smokers: Similarities and differences across commodity, sign, and magnitude. Journal of Abnormal Psychology, 112, 382-392.

BENZION, U., RAPOPORT, A., \& YAGIL, J. (1989). Discount rates inferred from decisions: An experimental study. Management Science, 35, 270-284.

Chapman, G. B. (1996). Temporal discounting and utility for health and money. Journal of Experimental Psychology: Learning, Memory, \& Cognition, 22, 771-791.

Chapman, G. B., \& Elstein, A. S. (1995). Valuing the future: Temporal discounting of health and money. Medical Decision Making, 15, 373-386.

Du, W., Green, L., \& Myerson, J. (2002). Cross-cultural comparisons of discounting delayed and probabilistic rewards. Psychological Record, 52, 479-492.

EsTES, W. K. (1956). The problem of inference from curves based on group data. Psychological Bulletin, 53, 134-140.

Federal Reserve Statistical Release G.i 9: Consumer Credit (2004). Retrieved May 11, 2004 from www.federalreserve.gov/ releases/G19/current/.

Frederick, S., Loewenstein, G., \& O'Donoghue, T. (2002). Time discounting and time preference: A critical review. Journal of Economic Literature, 40, 351-401.

Gonzalez, R., \& Wu, G. (1999). On the shape of the probability weighting function. Cognitive Psychology, 38, 129-166.

Green, L., Fry, A. F., \& Myerson, J. (1994). Discounting of delayed rewards: A life-span comparison. Psychological Science, 5, 33-36.

Green, L., \& Myerson, J. (1996). Exponential versus hyperbolic discounting of delayed outcomes: Risk and waiting time. American $\mathrm{Zo}-$ ologist, 36, 496-505.

Green, L., \& Myerson, J. (2004). A discounting framework for choice with delayed and probabilistic rewards. Psychological Bulletin, 130, 769-792

Green, L., Myerson, J., \& McFadden, E. (1997). Rate of temporal discounting decreases with amount of reward. Memory \& Cognition, 25, 715-723.

Green, L., Myerson, J., \& Ostaszewski, P. (1999). Amount of reward has opposite effects on the discounting of delayed and probabilistic outcomes. Journal of Experimental Psychology: Learning, Memory, \& Cognition, 25, 418-427.

Holt, D. D., Green, L., \& Myerson, J. (2003). Is discounting impulsive? Evidence from temporal and probability discounting in gambling and non-gambling college students. Behavioural Processes, 64, 355-367.

Kahneman, D., \& Tversky, A. (1979). Prospect theory: An analysis of decision under risk. Econometrica, 47, 263-292.

KIRBY, K. N. (1997). Bidding on the future: Evidence against normative discounting of delayed rewards. Journal of Experimental Psychology: General, 126, 54-70

Kirby, K. N., \& Maraković, N. N. (1995). Modeling myopic decisions: Evidence for hyperbolic delay-discounting within subjects and amounts. Organizational Behavior \& Human Decision Processes, 64, 22-30.

LAIBSON, D. (1997). Golden eggs and hyperbolic discounting. Quarterly Journal of Economics, 11, 443-477.

Loewenstein, G. (1992). The fall and rise of psychological explanations in the economics of intertemporal choice. In G. Loewenstein \& J. Elster (Eds.), Choice over time (pp. 3-34). New York: Sage.

Loewenstein, G., \& Prelec, D. (1992). Anomalies in intertemporal choice: Evidence and an interpretation. Quarterly Journal of Economics, 107, 573-597.

Loewenstein, G., \& Thaler, R. H. (1989). Intertemporal choice. Journal of Economic Perspectives, 3, 181-193.

MAZUR, J. E. (1987). An adjusting procedure for studying delayed reinforcement. In M. L. Commons, J. E. Mazur, J. A. Nevin, \& H. Rachlin (Eds.), Quantitative analyses of behavior: Vol. 5. The effect of delay and of intervening events on reinforcement value (pp. 55-73). Hillsdale, NJ: Erlbaum.

Murphy, J. G., Vuchinich, R. E., \& Simpson, C. A. (2001). Delayed reward and cost discounting. Psychological Record, 51, 571-588.

Myerson, J., \& Green, L. (1995). Discounting of delayed rewards: Models of individual choice. Journal of the Experimental Analysis of Behavior, 64, 263-276.

Myerson, J., Green, L., Hanson, J. S., Holt, D. D., \& Estle, S. J. (2003). Discounting delayed and probabilistic rewards: Processes and traits. Journal of Economic Psychology, 24, 619-635.

Myerson, J., Green, L., \& Warusawitharana, M. (2001). Area under the curve as a measure of discounting. Journal of the Experimental Analysis of Behavior, 76, 235-243.

Prelec, D., \& Loewenstein, G. (1991). Decision making over time and under uncertainty: A common approach. Management Science, 37, 770-786.

Rachlin, H., Logue, A. W., Gibbon, J., \& Frankel, M. (1986). Cognition and behavior in studies of choice. Psychological Review, 93, 33-45.

Rachlin, H., Raineri, A., \& Cross, D. (1991). Subjective probability and delay. Journal of the Experimental Analysis of Behavior, 55, 233244

Rachlin, H., Siegel, E., \& Cross, D. (1994). Lotteries and the time horizon. Psychological Science, 5, 390-393.

RAINERI, A., \& RACHLIN, H. (1993). The effect of temporal constraints on the value of money and other commodities. Journal of Behavioral Decision Making, 6, 77-94.

SidMAN, M. (1952). A note on functional relations obtained from group data. Psychological Bulletin, 49, 263-269.

Simpson, C. A., \& Vuchinich, R. E. (2000). Reliability of a measure of temporal discounting. Psychological Record, 50, 3-16.

STARMER, C. (2000). Developments in non-expected utility theory: The hunt for a descriptive theory of choice under risk. Journal of Economic Literature, 38, 332-382.

Stevens, S. S. (1957). On the psychophysical law. Psychological Review, 64, 153-181.

Stevenson, M. K. (1986). A discounting model for decisions with delayed positive and negative outcomes. Journal of Experimental Psychology: General, 115, 131-154.

THALER, R. (1981). Some empirical evidence on dynamic inconsistency. Economics Letters, 8, 201-207.

Tversky, A., \& Kahneman, D. (1981). The framing of decisions and the psychology of choice. Science, 211, 453-458.

TVERSKY, A., \& KAHNEMAN, D. (1992). Advances in prospect theory: Cumulative representation of uncertainty. Journal of Risk \& Uncertainty, 5, 297-323.

U.S. Census Bureau (2003). Statistical Abstract of the United States: 2003 (123rd ed.). Washington, DC: U.S. Government Printing Office.

Yates, J. F., \& Watts, R. A. (1975). Preferences for deferred loss. Organizational Behavior \& Human Performance, 13, 294-306.

\section{NOTE}

1. The average household credit card debt was calculated by dividing the total amount of revolving consumer debt in the United States at the end of 2002 (Federal Reserve Statistical Release G.19: Consumer Credit, May 7, 2004) by the number of households in 2002 (U.S. Census Bureau, 2003).

\section{ARCHIVED MATERIALS}

The following materials associated with this article may be accessed through the Psychonomic Society's Norms, Stimuli, and Data archive, www.psychonomic.org/archive. 
To access these files, search the archive for this article using the journal name (Memory \& Cognition), the first author's name (Estle), and the publication year (2006).

FILE: Estle-M\&C-2006.zip

DESCRIPTION: The compressed archive file contains five files:

Estle-M\&C-2006.xls, containing the raw data (subjective value of the delayed/probabilistic outcome at each of the delays/probabilities for each of the four experiments), as a $46.5 \mathrm{~K}$ Excel file. The Excel file contains a separate worksheet for each experiment. Each row represents a different participant; each column the subjective value obtained for a particular delay/probability at a particular amount. The first row contains labels for each of the columns.

Estle-M\&C-2006-exp1.txt, Estle-M\&C-2006-exp2.txt, Estle-M\&C2006-exp3.txt, and Estle-M\&C-2006-exp4.txt, containing the raw data for Experiments 1, 2, 3, and 4 as $4 \mathrm{~K}, 3 \mathrm{~K}, 8 \mathrm{~K}$, and $7 \mathrm{~K}$ tab-delimited text files, respectively.

AUTHOR's E-MAIL ADDRESs: 1green@wustl.edu.

(Manuscript received February 15, 2005; revision accepted for publication May 17, 2005.) 